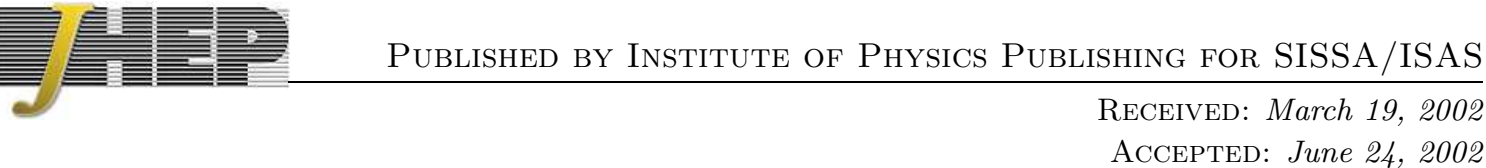

ACCEPTED: June 24, 2002

\title{
Cosmological perturbations and short distance physics from noncommutative geometry
}

\author{
Fedele Lizzi, Gianpiero Mangano, Gennaro Miele \\ Dipartimento di Scienze Fisiche, Università di Napoli Federico II \\ and INFN, Sezione di Napoli, \\ Monte S. Angelo, Via Cintia, 80126 Napoli, Italy \\ E-mail: fedele.lizzi@na.infn.it, gianpiero.mangano@na.infn.it, \\ gennaro.miele@na.infn.it \\ Marco Peloso \\ Physikalisches Institut, Universität Bonn \\ Nussallee 12, D-53115 Bonn, Germany \\ E-mail: peloso@th.physik.uni-bonn.de
}

\begin{abstract}
We investigate the possible effects on the evolution of perturbations in the inflationary epoch due to short distance physics. We introduce a suitable non local action for the inflaton field, suggested by Noncommutative Geometry, and obtained by adopting a generalized star product on a Friedmann-Robertson-Walker background. In particular, we study how the presence of a length scale where spacetime becomes noncommutative affects the gaussianity and isotropy properties of fluctuations, and the corresponding effects on the Cosmic Microwave Background spectrum.
\end{abstract}

Keywords: Non-Commutative Geometry, Cosmology of Theories beyond the SM. 


\section{Contents}

1. Introduction: noncommutative spaces and deformed products

2. Noncommuting variables in the inflation era 3

3. Nonlocal free scalar field in an expanding universe 5

4. A possible signature in the cosmic microwave background 8

5. Conclusions 12

\section{Introduction: noncommutative spaces and deformed products}

The increasing precision of astronomical data is opening the possibility that the study of several features of our universe may give hints on yet unknown properties of fundamental interactions at energy scales well beyond the domain of standard accelerator experiments. For example, it is conceivable that some cosmological observables, influenced by the very early stages of the evolution of the universe, may provide clues on the nature of gravitational interactions at very small distances. Although it is standard to assume that the scale at which the properties of spacetime are modified is close to the exceedingly small Planck length $l_{P}$, many models have been recently proposed in which this scale (set presumably by the fundamental string length) is several order of magnitude higher [1, 2, 3].

Inflation provides a particularly suitable framework for these considerations, through its predictions for the primordial perturbations. These perturbations, responsible for structure formation and for the temperature anisotropies in the Cosmic Microwave Background (CMB), arise as quantum fluctuations during the inflationary epoch and are stretched to cosmological scales by the huge expansion. Hence, they are sensitive to physics at distances at least as small as the horizon size during inflation, which in the simplest models is about five orders of magnitude higher than $l_{P}$, although they are probably generated at even smaller scales.

This issue has been studied in the last few years by several authors [4]-17] (other cosmological and astrophysical implications of new physics at short distance have also been discussed. See for example [18]-[21]). Although they use different models, most of these analyses share the idea that the appearance of this new length scale can be modelled in terms of a transition from an ordinary to a "quantum" spacetime, in a way which is reminiscent of the transition from classical to quantum phase space. Another possibility is to consider a deformation of the usual Heisenberg algebra [22, 23]. The general finding is that short distance physics may affect both the gaussian character of the fluctuations, as well as their isotropy. 
It has been recently realized [24, 25] that a consequence of string theory is that the structure of spacetime becomes noncommutative. The noncommutativity of the vertex operators, in the low momentum limit, representing the interaction of strings, and whose low energy limit gives rise to ordinary spacetime [26], leads to a spacetime which can be described loosely as an analog of a quantum phase space, in terms of the algebra generated by noncommuting coordinates

$$
\left[x^{\mu}, x^{\nu}\right]=i \Theta^{\mu \nu}(x),
$$

with $\Theta^{\mu \nu}$ an antisymmetric tensor. This is a particular kind of a Noncommutative Geometry [27, 28, 29, 30]. Since the coordinates do not commute, a Heisenberg principle is at work, and the concept of point is no more meaningful. This is similar to what happens in the quantization of a classical system. In this case the phase space loses its meaning as an ordinary manifold, and the observable quantities corresponding to the coordinates in phase space become selfadjoint elements of the algebra of operators on an Hilbert space. To each function on phase space is then associated an operator on an Hilbert space, and the product of functions is the operator product, which is noncommutative. An equivalent procedure 31] is to consider a deformation of the algebra of functions on the ordinary phase space. The functions are the same, but their product is not the usual pointwise one, but one which reproduces the multiplication of operators. The technique to study noncommutative spacetime is similar. The main tool is the definition of the deformed algebra. For a constant $\Theta^{\mu \nu}$, and in a flat space, the $\star$ product [32, 33] is defined as follows ${ }^{1}$

$$
\begin{aligned}
f \star g & \equiv \sum_{k=0}^{\infty} \frac{1}{k !}\left(\frac{i}{2}\right)^{k} \Theta^{\mu_{1} \nu_{1}} \cdots \Theta^{\mu_{k} \nu_{k}}\left(\partial_{\mu_{1}} \cdots \partial_{\mu_{k}} f\right)\left(\partial_{\nu_{1}} \cdots \partial_{\nu_{k}} g\right) \\
& =f g+\frac{i}{2} \Theta^{\mu \nu} \partial_{\mu} f \partial_{\nu} g-\frac{1}{8} \Theta^{\mu_{1} \nu_{1}} \Theta^{\mu_{2} \nu_{2}}\left(\partial_{\mu_{1}} \partial_{\mu_{2}} f\right)\left(\partial_{\nu_{1}} \partial_{\nu_{2}} g\right)+O\left(\Theta^{3}\right) .
\end{aligned}
$$

At zeroth order in $\Theta^{\mu \nu}$ this expression reduces to the ordinary (commutative) pointwise product while, at higher order, derivatives of the fields appear. The definition (1.2) generalizes eq. (1.1) in that $x^{\mu} \star x^{\nu}-x^{\nu} \star x^{\mu}=i \Theta^{\mu \nu}$. A theory on a noncommutative space of this kind is obtained by substituting all products between fields in the action by the $\star$ product. This implies that the theory becomes nonlocal.

Field theories with $\star$ products have been studied extensively, from the early work of 34, [35, 36, 37]. For recent reviews with extensive references see for example [38, 39, 40, 41]. Most of the work has been done in the case of flat spacetime, and for a constant $\Theta^{\mu \nu}$ with only space-like nonvanishing components, $\Theta^{0 i}=0$ (hereafter we use latin indexes for spatial components). The main motivation for this choice is that the theory would be local in time, and therefore unitary. On the other hand, in order to preserve Lorentz invariance, the commutator of the $x^{\mu}$ 's must be a tensor, and its transformation under a boost will produce nonvanishing space-time components. This problem has been investigated in 42, 43] among others. The solution to this puzzle lies in the fact that a theory described by a $\star$ product,

\footnotetext{
${ }^{1}$ If $\Theta^{\mu \nu}$ is non degenerate, the product in 1.2 is an asymptotic expansion of the more general integral expression $(f \star g)(x)=(1 / \pi)^{n} \int_{\mathbb{R}^{n}} \int_{\mathbb{R}^{n}} \exp \left(2 i\left(\Theta^{-1}\right)_{\mu \nu}\left(x^{\mu} y^{\nu}+y^{\mu} z^{\nu}+z^{\mu} x^{\nu}\right)\right) f(y) g(z) d^{n} y d^{n} z$. For a primer of the various expressions of the product see [30, section 3.5].
} 
as we said, is only an effective theory of a more fundamental theoretical framework, which only in a low energy limit gives rise to the relatively simple product (1.2). In string theory the components $\Theta^{i j}$ correspond to a magnetic field, while the $\Theta^{0 i}$ to an electric field. A treatment of scattering of strings in an electric field 44, 45] shows that in this case unitarity is restored by the presence of massive open strings modes, or branes, which cannot be neglected. In light of these results the unitarity condition can be cast in an invariant form, by requiring

$$
\Theta^{\mu \nu} \Theta_{\mu \nu}>0, \quad \epsilon_{\mu \nu \rho \sigma} \Theta^{\mu \nu} \Theta^{\rho \sigma}=0,
$$

i.e. there exists a particular frame where only space-like components of $\Theta^{\mu \nu}$ are nonvanishing. In all other frames brane modes should be included to guarantee a unitary behaviour of the dynamics. Another possibility [46], which does not make use of string theory, is based on the framework developed in [34]. In the rest of the paper we will take $\Theta^{\mu \nu}$ to have only space-space nonvanishing components.

The $\star$ product defined in eq. (1.2) defines an associative algebra for a constant $\Theta^{\mu \nu}$, and flat Minkowski metric. The problem of finding a deformed product, in the most general case of a Poisson manifold, has been formally solved by Kontsevich [47. This solution is however at the level of formal series in the coordinates. Hence, although of great interest from a mathematical point of view, it is of limited practical use. Other noncommutative geometries leading to deformed products have been discussed in various contexts, for example in a class of deformed products based on three dimensional Lie algebras [48], but the list of examples is rather lengthy [49, 50, 51]. All of these products are nonlocal.

In this paper we discuss some effects that a nonlocal theory, based on noncommutative geometry, might have on the evolution of perturbations produced during the inflationary epoch. As in [14], we will start considering a $\star$ deformed action for a scalar field at the lowest non trivial order in $\Theta^{\mu \nu}$. However, in the spirit of general covariance, we first introduce in section 2 a suitable covariant definition for star product, which reduces to (1.2) in flat space, and discuss our assumptions on the antisymmetric tensor $\Theta^{\mu \nu}$. We then consider the simplest case of a quadratic potential for the inflaton field, though the formalism can be straightforwardly applied to an arbitrary polynomial potential. In section 3 we deduce the equation of motion for field fluctuations, which can be cast in a simple form, analogous to the usual commutative case, up to a term which breaks spatial isotropy. Finally we consider how our results may affect the CMB spectrum, and give our conclusions and outlooks.

\section{Noncommuting variables in the inflation era}

We assume that the effect of a quantum-like behaviour of spacetime at short distances can be described via the introduction of the non trivial commutator (1.1) for comoving coordinates $x^{\mu} \equiv(t, \mathbf{x})$ for a flat Friedmann-Robertson-Walker (FRW) metric. ${ }^{2}$ There are some natural requirements which limit the choice of the dimensionful quantity $\Theta^{\mu \nu}$ :

\footnotetext{
${ }^{2}$ We use unit $\hbar=c=1$.
} 
(i) to satisfy unitarity in the comoving frame we take $\Theta^{0 i}=0$;

(ii) we require that the uncertainty principle, which follows from eq. (1.1), affects localization in physical coordinates only at some length scale, and is therefore consistent with a correspondence principle. The simplest form for $\Theta^{i j}$ which realizes this condition in the comoving frame is the following

$$
\Theta^{i j}=\frac{f^{i j}(\mathbf{x})}{a^{2}},
$$

where $a$ is the scale factor of the universe and its exponent in (2.1) is dictated by the above condition;

(iii) in a homogenous background metric, it is reasonable to assume a spatially constant $\Theta^{\mu \nu}$. With no loss of generality we can therefore choose a frame where the only nonvanishing space-space component of $\Theta^{\mu \nu}$ is

$$
\Theta^{12}(t)=\frac{1}{\Lambda^{2} a^{2}}
$$

with $\Lambda^{-1}$ the noncommutativity length scale.

It is interesting to note that, using the expressions for the non vanishing Levi Civita connections in a critical FRW spacetime

$$
\Gamma_{0 j}^{i}=\frac{\dot{a}}{a} \delta_{j}^{i}, \quad \Gamma_{i j}^{0}=a \dot{a} \delta_{i j},
$$

expression (2.2) implies

$$
D_{\rho} \Theta^{12}=0
$$

with $D_{\rho}$ the covariant derivative.

We now construct a covariant, nonlocal action for a scalar field $\phi$, inspired by noncommutative geometry, with the introduction of a deformed $*$ product $^{3}$

For a polynomial potential the effective action we will consider will be of the form

$$
\begin{aligned}
S & =\int d^{4} x \sqrt{-g}\left[\frac{1}{2}\left(\partial_{\mu} \phi\right) *\left(\partial^{\mu} \phi\right)-\frac{\lambda}{n !} \phi * \cdots * \phi\right] \\
& \equiv \int d^{4} x \sqrt{-g}\left[\frac{1}{2}\left(\partial_{\mu} \phi\right) *\left(\partial^{\mu} \phi\right)-\frac{\lambda}{n !} \phi^{* n}\right],
\end{aligned}
$$

Since spacetime is now curved, we cannot unambiguously use the $\star$ product defined in (1.2) since it is not manifestly covariant. This however immediately suggests that the simplest generalization is via the substitution of ordinary partial derivatives in (1.2) with covariant derivatives. We therefore define a new, curved, $*$ product

$$
f * g \equiv \sum_{k=0}^{\infty} \frac{1}{k !}\left(\frac{i}{2}\right)^{k} \Theta^{\mu_{1} \nu_{1}} \cdots \Theta^{\mu_{k} \nu_{k}}\left(D_{\mu_{1}} \cdots D_{\mu_{k}} f\right)\left(D_{\nu_{1}} \cdots D_{\nu_{k}} g\right) .
$$

\footnotetext{
${ }^{3}$ We will reserve the symbol $\star$ for the flat case product defined in (1.2), with a constant $\Theta^{\mu \nu}$.
} 
The main problem with the definition (2.6) is that it defines a product, in general, nonassociative. In flat space, covariant derivatives reduce to commuting ordinary derivatives, and with a constant $\Theta^{\mu \nu}$, the product $\star$ is associative. In a curved background, the constancy requirement translates into the condition that $D_{\rho} \Theta^{\mu \nu}=0$ for all non vanishing components which appear in (2.6). Since the solution (2.2) satisfies this requirement, this further justifies our choice.

However the appearance of the commutators $\left[D_{\mu}, D_{\nu}\right]$, which in a curved background vanish only on scalar functions spoils associativity. Notice that (thanks to the choice (2.2)) the nonassociativity appears only at fourth order in $\Theta^{\mu \nu}$, while noncommutative effects are already present at order $\Theta^{2}$. This enable us to apply a kind of $\Theta^{\mu \nu}$-perturbative analysis to any analytic potential $V=\sum_{n} \lambda_{n} \phi^{* n}$, as well as to evaluate scalar field $n$ point functions, $\left\langle\phi\left(t, \mathbf{x}_{\mathbf{1}}\right) * \cdots * \phi\left(t, \mathbf{x}_{\mathbf{n}}\right)\right\rangle$, provided we only consider the lowest nontrivial (quadratic) contribution in $\Theta^{\mu \nu}$. In the following we will spell out the conditions under which this is justified.

To conclude this section we note, in passing, that the algebra of the vertex operators representing fundamental ground state strings is only associative in the limit of the string parameter $\alpha^{\prime} \rightarrow 0$ [25]. Other nonassociative products (of a different kind) have appeared in the connections between strings and noncommutative geometry 52].

\section{Nonlocal free scalar field in an expanding universe}

We consider the action (2.5) for a quadratic potential

$$
S=\int d^{4} x \sqrt{-g}\left[\frac{1}{2}\left(\partial_{\mu} \phi\right) *\left(\partial^{\mu} \phi\right)-\frac{m^{2}}{2} \phi * \phi\right]=S_{0}+\delta S_{K}+\delta S_{V}+\mathcal{O}\left(\Theta^{4}\right),
$$

where $\delta S_{K}$ and $\delta S_{V}$ represent the lowest non trivial corrections $\left(\mathcal{O}\left(\Theta^{2}\right)\right)$ to the kinetic and potential terms in $S$ respectively. The correction to the mass term can be rewritten as ${ }^{4}$

$$
\begin{aligned}
\delta S_{V} & =-\frac{m^{2}}{32} \int d^{4} x \sqrt{-g} \Theta^{\mu \nu} \Theta^{\rho \sigma}\left(\left[D_{\mu}, D_{\nu}\right] D_{\sigma} \phi\right) D_{\rho} \phi= \\
& =\frac{m^{2}}{32} \int d^{4} x \sqrt{-g} \Theta^{\mu \nu} \Theta^{\rho \sigma} R_{\sigma \mu \nu}^{\tau} \partial_{\tau} \phi \partial_{\rho} \phi .
\end{aligned}
$$

To obtain this expression, we have used that $D_{\mu} \Theta^{12}=0$ and assumed the vanishing of the boundary term

$$
\begin{aligned}
\int d^{4} x \sqrt{-g} D_{\mu} V^{\mu} & =\int d^{4} x \partial_{\mu}\left(a^{3} V^{\mu}\right) \\
V^{\mu} & \equiv \Theta^{\mu \nu} \Theta^{\rho \sigma} D_{\rho} \phi\left(D_{\nu} D_{\sigma} \phi\right) .
\end{aligned}
$$

After some algebra and using the expressions for the Riemann tensor in a FRW universe, eq. (3.3) can be cast in the form

$$
\delta S_{V}=-\frac{m^{2}}{16} \int d^{4} x a^{3} \frac{1}{\Lambda^{4}}\left(\frac{\dot{a}}{a}\right)^{2}\left(\partial_{1} \phi \partial^{1} \phi+\partial_{2} \phi \partial^{2} \phi\right),
$$

\footnotetext{
${ }^{4}$ We adopt the notation of $[53$.
} 
With an analogous computation, one finds

$$
\begin{aligned}
\delta S_{K}=\frac{1}{32} \int d^{4} x \sqrt{-g} \Theta^{\mu \nu} \Theta^{\rho \sigma}\left(D_{\rho} D_{\tau} \phi\right)\left(\left[D_{\mu}, D_{\nu}\right] D_{\sigma} D^{\tau} \phi\right) & \\
=\frac{1}{16} \int d^{4} x a^{3} \frac{1}{\Lambda^{4}}\left(\frac{\dot{a}}{a}\right)^{2}[ & \partial_{m} \partial_{0} \phi \partial^{m} \partial^{0} \phi+\partial_{m} \partial_{i} \phi \partial^{m} \partial^{i} \phi- \\
& \left.-2 \frac{\dot{a}}{a} \partial^{m} \phi \partial_{0} \partial_{m} \phi+\left(\frac{\dot{a}}{a}\right)^{2} \partial_{m} \phi \partial^{m} \phi\right],
\end{aligned}
$$

with $m=1,2$.

The two terms $\delta S_{K}$ and $\delta S_{V}$ break isotropy. However, as long as $S_{0}$ is the leading contribution, the scalar field $\phi$ can be still assumed to be spatially constant up to small quantum fluctuations. Note that, for our choice of $\Theta^{\mu \nu}$, the corrections $\delta S$ do not contribute to the action for the homogeneous component. For this reason, the background evolves as in the standard case, if the backreaction of fluctuations can be neglected. To study the evolution of fluctuations, it is standard [54] to use conformal time $d \eta=d t / a$, and decompose

$$
\phi=\frac{\chi_{0}(\eta)}{a}+\int \frac{d^{3} \mathbf{k}}{(2 \pi)^{3 / 2} a}\left(\mathbf{a}_{\mathbf{k}} \chi_{\mathbf{k}}(\eta) \mathrm{e}^{i \mathbf{k} \cdot \mathbf{x}}+\mathbf{a}_{\mathbf{k}}^{\dagger} \chi_{\mathbf{k}}^{*}(\eta) \mathrm{e}^{-i \mathbf{k} \cdot \mathbf{x}}\right),
$$

with $\mathbf{a}_{\mathbf{k}}, \mathbf{a}_{\mathbf{k}}^{\dagger}$ satisfying canonical commutation relations. The action for fluctuations, up to second order, evaluated in the vacuum after normal ordering, becomes

$$
\begin{aligned}
S=\int d \eta d^{3} \mathbf{k}\left[\left|\chi_{\mathbf{k}}^{\prime}\right|^{2}+\left(\frac{a^{\prime \prime}}{a}-a^{2} m^{2}-k^{2}\right)\left|\chi_{\mathbf{k}}\right|^{2}+\right. & \\
+\frac{H^{2}}{8 \Lambda^{4} a^{2}}( & -k_{\perp}^{2}\left|\chi_{\mathbf{k}}^{\prime}\right|^{2}+k^{2} k_{\perp}^{2}\left|\chi_{\mathbf{k}}\right|^{2}+ \\
& \left.\left.+\left(10 a^{2} H^{2}-6 \frac{a^{\prime \prime}}{a}+a^{2} m^{2}\right) k_{\perp}^{2}\left|\chi_{\mathbf{k}}\right|^{2}\right)\right],
\end{aligned}
$$

where $k^{2} \equiv \sum_{i} k_{i}^{2}, k_{\perp}^{2} \equiv k_{1}^{2}+k_{2}^{2}, H=\dot{a} / a$, and ' denotes derivation with respect to $\eta$.

Since we are interested in the dynamics during the inflationary epoch, we consider a de Sitter Universe, ${ }^{5}$ setting the origin of time so that $\eta=-1 /(a H)$. It is convenient to redefine

$$
\begin{aligned}
\chi_{\mathbf{k}}(\eta) & \equiv\left(1-\epsilon^{2} k_{\perp}^{2} \eta^{2}\right)^{-1 / 2} y_{\mathbf{k}}(\eta), \\
\epsilon^{2} & \equiv \frac{H^{4}}{8 \Lambda^{4}},
\end{aligned}
$$

so that, up to a total derivative, the action (3.7) becomes

$$
\begin{aligned}
S & =\int d \eta d^{3} \mathbf{k}\left(\left|y_{\mathbf{k}}^{\prime}\right|^{2}-\omega_{\mathbf{k}}^{2}\left|y_{\mathbf{k}}\right|^{2}\right), \\
\omega_{\mathbf{k}}^{2} & \equiv k^{2}-\frac{2}{\eta^{2}}+\frac{m^{2}}{H^{2} \eta^{2}}+\frac{\epsilon^{2} k_{\perp}^{2}}{\left(1-\epsilon^{2} k_{\perp}^{2} \eta^{2}\right)^{2}},
\end{aligned}
$$

\footnotetext{
${ }^{5}$ In the model we are considering, $H$ is actually slowly decreasing, so that the spacetime is not exactly de Sitter. This leads to a small logarithmic decrease of the spectrum of fluctuations with increasing momentum [54], which for the purposes of this paper can be neglected.
} 
Notice that eq. (3.9) is meaningful only to first order in $\epsilon^{2} k_{\perp}^{2} \eta^{2}$, since we have neglected higher order corrections in $\Theta^{\mu \nu}$ in the action (3.1). Our perturbative analysis is thus valid only for

$$
\eta^{2}<\eta_{i}^{2} \equiv \frac{8 \Lambda^{4}}{H^{4} k^{2}}
$$

This condition can be easily understood as follows. In a de Sitter Universe, physical scales $a / k$ grow exponentially, while the horizon $H^{-1}$ is constant. Any given mode $k$ is then well inside the horizon in the remote past $(\eta \rightarrow-\infty)$ and well outside in the remote future $(\eta \rightarrow 0)$. For our choice of the time origin, the horizon crossing occurs at $\eta_{c}=-1 / k$. If we rewrite eq. (3.10) as

$$
\frac{a / k}{\Lambda^{-1}}>\left(\frac{H^{2}}{8 \Lambda^{2}}\right)^{1 / 2},
$$

we see that our analysis can be applied to a given mode $k$ only for times such that the corresponding physical scale is larger than the length scale $\epsilon^{1 / 2} \Lambda^{-1}$. Imposing $\eta_{i}<\eta_{c}$ so that we can follow the dynamical evolution of the mode $k$ at quadratic order in $\Theta^{\mu \nu}$ well before its horizon crossing, simply amounts to require that $\Lambda>H$, i.e. that the scale of noncommutativity is smaller than the size of the horizon. Of course this result also shows that any new effect induced by the length scale $\Lambda^{-1}$ is not negligible provided this parameter is not much smaller than the horizon size, [5, 6, 11].

For $\eta \ll \eta_{i}$, the equation of motion following from eq. (3.9) becomes $^{6}$

$$
y_{\mathbf{k}}^{\prime \prime}+\left(-\frac{2}{\eta^{2}}+k^{2}+\epsilon^{2} k_{\perp}^{2}\right) y_{\mathbf{k}}=0 .
$$

In the time interval $\eta_{i} \ll \eta \ll-1 / k$, the action of a given mode $y_{\mathbf{k}}$ is approximately the one of a harmonic oscillator with the adiabatically changing frequency $\omega_{\mathbf{k}}$. Condition $\eta \ll-1 / k$ indicates that the mode is well inside the horizon, so that the expansion of the Universe is at this stage negligible. Since the frequency is adiabatically changing, we adopt the standard procedure (see for example [54]) to start with the adiabatic vacuum state

$$
y_{\mathbf{k}}=\frac{1}{\sqrt{2 \omega_{\mathbf{k}}}} \mathrm{e}^{-i \omega_{\mathbf{k}} \eta} .
$$

As well known, the choice of initial conditions is a major problem. In the standard scenario one assumes that modes emerge at the Planck scale from a stage where quantum gravity effects cannot be ignored. Subsequent evolution of these modes is then ruled by known physics. In our analysis, the threshold scale is given by $\eta=\eta_{i}$, i.e. for an initial physical scale of the mode of the order of $H / \Lambda^{2}$. If during these early times the frequency of any given mode is real and adiabatically changing $\left(\omega^{\prime} \ll \omega^{2}\right)$, one expects that fluctuations are always "kept" in the adiabatic vacuum (3.13), since this minimizes the energy of the field (see, for example, [5] for a recent discussion). Imposing $\eta_{i} \ll-1 / k$ allows us to have an adiabatically changing frequency for a nonvanishing time interval, from which the choice (3.13) is motivated. Although eq. (3.9) may lead to the conclusion that the

\footnotetext{
${ }^{6}$ We neglect the mass term contribution to $\omega_{\mathbf{k}}^{2}$, see eq. 3.9 ). From a more detailed analysis (i.e. taking into account the time dependence of $H$ ), one can show that this term is relevant only at the end of inflation.
} 
adiabaticity condition breaks at $\eta \simeq \eta_{i}$, we stress that this equation is valid for $\eta \gg \eta_{i}$, so that we cannot exclude on the basis of the present analysis that the change of frequency is indeed adiabatic also at earlier times.

We thus start from (3.13) and solve the evolution equation (3.12). Expanding also the redefinition (3.8) to first order in $\epsilon^{2} k_{\perp}^{2} \eta^{2}$, one finds

$$
\begin{aligned}
\varphi_{\mathbf{k}} & \equiv \frac{\chi_{\mathbf{k}}}{a}=\frac{H \eta}{\sqrt{2 \alpha k}} \mathrm{e}^{-i \alpha k \eta}\left(1-\frac{1}{2}\left(\alpha^{2}-1\right) k^{2} \eta^{2}\right)\left[1-\frac{i}{\alpha k \eta}\right], \\
\alpha & \equiv \sqrt{1+\epsilon^{2} \sin ^{2} \vartheta}
\end{aligned}
$$

where $k_{\perp}=k \sin \vartheta$ and $\vartheta$ is the angle between $k$ and the third axis. From this result and eq. (3.8), the scalar fluctuation have the late time amplitude

$$
\left|\varphi_{\mathbf{k}}\right|=\frac{H}{\sqrt{2} k^{3 / 2}}\left(1-\frac{3}{32} \frac{H^{4}}{\Lambda^{4}} \sin ^{2} \vartheta\right)
$$

up to correction of higher order in $\epsilon^{2}$.

The first term in (3.15) is the standard result, valid for fluctuations of a massless field in a de Sitter universe. The second is instead a new effect induced by the $\Theta^{\mu \nu}$ depending terms in the action (3.1), and explicitly shows the presence of a preferred direction associated with the nonvanishing component $\Theta^{12}$. It is worth noticing that this correction does not decrease at later times, so that the imprint of noncommutativity is preserved even after the physical scales of the fluctuations have grown to much larger sizes than $\Lambda^{-1}$. In the next section, we will discuss a possible observational implication of this result.

\section{A possible signature in the cosmic microwave background}

One of the main successes of inflation is the generation of a nearly flat spectrum of adiabatic scalar metric perturbations. These primordial inhomogeneities are the seeds of both structure formation and the temperature anisotropies observed in the CMB . In this section we discuss how our findings may affect these perturbations.

We first briefly review the standard case. For an isotropic stress energy tensor, as it is the case for a minimally coupled scalar field, scalar metric perturbations can be described by a single gauge invariant variable $\Phi$ [55]. In longitudinal gauge, the perturbed metric can be written in terms of $\Phi$ as

$$
d s^{2}=d t^{2}(1+2 \Phi)-a^{2} d \mathbf{x}^{2}(1-2 \Phi)
$$

For a semiclassical treatment of fluctuations, necessary in order to find their initial nor-

malization, it is convenient to introduce a second gauge invariant quantity [56], which is a superposition of the scalar metric fluctuation $\Phi$ and the inflaton fluctuation $\varphi$

$$
v=a\left(\varphi+\frac{z}{a} \Phi\right), \quad z \equiv \frac{a \dot{\phi}}{H}
$$


Using Einstein equations, the action for fluctuations up to second order can be written in a remarkably simple form for $v_{k}$, the Fourier transform of $v$

$$
S=\frac{1}{2} \int d \eta d^{3} \mathbf{k}\left[\left|v_{k}^{\prime}\right|^{2}-k^{2}\left|v_{k}\right|^{2}+\frac{z^{\prime \prime}}{z}\left|v_{k}\right|^{2}\right] .
$$

This expression further simplifies during inflation, due to the slow motion of the inflaton field. For inflation to occur, the $\phi$ field must be in a region of the potential which satisfies the flatness conditions

$$
\begin{aligned}
& \tilde{\varepsilon} \equiv \frac{M_{P}^{2}}{2}\left(\frac{1}{V} \frac{\delta V}{\delta \phi}\right)^{2} \ll 1, \\
& \tilde{\eta} \equiv M_{P}^{2} \frac{1}{V} \frac{\delta^{2} V}{\delta \phi^{2}} \ll 1,
\end{aligned}
$$

where $M_{P}$ is the reduced Planck mass $M_{P}=2.4 \cdot 10^{18} \mathrm{GeV}$. As long as the slow roll conditions (4.4) hold, one finds

$$
z^{\prime} \simeq z \frac{a^{\prime}}{a}(1-\tilde{\eta}+2 \tilde{\varepsilon}) \simeq z \frac{a^{\prime}}{a} .
$$

The action (4.3) thus simplifies to

$$
S=\frac{1}{2} \int d \eta d^{3} \mathbf{k}\left[\left|v_{k}^{\prime}\right|^{2}-k^{2}\left|v_{k}\right|^{2}+\frac{a^{\prime \prime}}{a}\left|v_{k}\right|^{2}\right],
$$

which coincides with the action of a (properly normalized) massless scalar field, see eq. (3.7) with vanishing $\Theta^{\mu \nu}$.

In the following we want to study how the effects discussed in the previous section may modify this standard picture. Admittedly, these considerations cannot be completely rigorous, since it is likely that also the fluctuations of the field $\Theta^{\mu \nu}(t)$ should be included in the calculation. This would amount in considering the (unknown) dynamics of $\Theta^{\mu \nu}$.

On the other hand, we stress that, at least in the standard case, the possibility to describe metric scalar fluctuations as the fluctuations of a massless minimally coupled scalar field is mainly due to the slow evolution of the inflaton field, i.e. to the slow roll conditions (4.4), which (as all background quantities) are left unchanged by the choice $\Theta^{0 i}=0$. We thus may proceed in analogy with the standard case, and assume that eq. (3.7), with $m=0$, describes also the dynamics of the fluctuations $v_{k}$. In this way, we follow the common procedure (see e.g. [4]- [17]) to assume that nonstandard physics modifies the dispersion relation of quantum field excitations, and to use this relation (in our case the frequency $\omega_{\mathbf{k}}^{2}$ given in eq. (3.9)) to compute also the evolution of metric perturbations. From the analysis of the previous section, we find (see eq. (3.14))

$$
v_{\mathbf{k}}=-\frac{-i H}{\sqrt{2}} \frac{\mathrm{e}^{-i \alpha k \eta}}{(\alpha k)^{3 / 2}}\left(1-\frac{1}{2}\left(\alpha^{2}-1\right) k^{2} \eta^{2}\right)(1+i \alpha k \eta)
$$

From $v_{\mathbf{k}}$ one can define another gauge invariant quantity (originally denoted as $\phi_{m}$ in [55], and as $\mathcal{R}$ in [57) $\zeta_{\mathbf{k}} \equiv H v_{\mathbf{k}} /(a \dot{\phi})$ which represents the metric scalar perturbation on comoving hypersufaces, on which fluctuations of the stress energy tensor are at rest. Except 
on scales where gravitational collapse has taken place, this primordial quantity is related to the observed CMB temperature anisotropies $\delta T / T$ through a linear transfer function $\mathcal{T}$. This function depends upon physics at scales which are much larger than the scale of noncommutativity $\Lambda^{-1}$, and therefore it is perfectly legitimate to assume that $\mathcal{T}$ is rotationally invariant as in the standard case. Hence, by expanding the temperature anisotropies in terms of spherical harmonics $Y_{l m}$, one can write [57]

$$
\begin{aligned}
\frac{\delta T}{T} & =\sum_{l=1}^{\infty} \sum_{m=-l}^{l} a_{l m} Y_{l m}, \\
a_{l m} & =\frac{4 \pi i^{l}}{(2 \pi)^{3}} \int d^{3} \mathbf{k} \mathcal{T}(k, l) \zeta_{\mathbf{k}} Y_{l m}(\hat{\mathbf{k}}) .
\end{aligned}
$$

The first issue to discuss is whether the effects of noncommutativity may produce temperature fluctuations which deviate from a gaussian distribution. We start introducing the equal time $n$-point correlation function for the gauge invariant quantity $v(\mathbf{x}, \eta)$

$$
\Delta\left(\mathbf{x}_{1}, \ldots, \mathbf{x}_{\mathbf{n}} ; \eta\right) \equiv\left\langle v\left(\mathbf{x}_{1}, \eta\right) * \cdots * v\left(\mathbf{x}_{\mathbf{n}}, \eta\right)\right\rangle .
$$

To compute this quantity we therefore need a generalized $*$ product definition acting on (scalar) functions evaluated at different spacetime points. In analogy with our discussion of section 2, it is easy to see that this can be defined, at quadratic order in $\Theta^{\mu \nu}$, as follows (see for example [38])

$$
\begin{aligned}
& f_{1}\left(\mathbf{x}_{1}, \eta\right) * \cdots * f_{n}\left(\mathbf{x}_{\mathbf{n}}, \eta\right) \equiv \\
& \quad \equiv\left(1+\frac{i}{2} \sum_{a<b} \Theta^{\mu \nu} D_{\mu}^{a} D_{\nu}^{b}-\frac{1}{8} \sum_{a<b, c<d} \Theta^{\mu \nu} \Theta^{\rho \sigma} D_{\mu}^{a} D_{\nu}^{b} D_{\rho}^{c} D_{\sigma}^{d}\right) f_{1}\left(\mathbf{x}_{\mathbf{1}}, \eta\right) \cdots f_{n}\left(\mathbf{x}_{\mathbf{n}}, \eta\right),
\end{aligned}
$$

where $a, b, c, d$ run over $1, \ldots, n$ and, as in section 2, the only non vanishing component is $\Theta^{12}$, satisfying $D_{\rho} \Theta^{12}=0$. Notice that since we are considering an equal time product, and $\Theta^{12}$ depends on $\eta$ only, this expression is defined unambiguously. Moreover, reasoning as in section 2, it is worth stressing that the product (4.10) is associative when applied to scalar functions, so that the $n$-point function (4.9) is uniquely defined and can be expressed, via Wick theorem, in terms of lower order correlation functions.

Using (4.10) and the definition of $\epsilon^{2}$ in (3.8), it is easy to get

$$
\begin{aligned}
\Delta\left(\mathbf{x}_{\mathbf{1}}, \mathbf{x}_{\mathbf{2}} ; \eta\right)= & \int \frac{d^{3} \mathbf{k}}{2 \pi^{3}} e^{i \mathbf{k} \cdot\left(\mathbf{x}_{\mathbf{1}}-\mathbf{x}_{\mathbf{2}}\right)}\left|v_{\mathbf{k}}\right|^{2}\left(1-2 \epsilon^{2} k^{2} \eta^{2}\right) \\
\Delta\left(\mathbf{x}_{\mathbf{1}}, \mathbf{x}_{\mathbf{2}}, \mathbf{x}_{\mathbf{3}}, \mathbf{x}_{\mathbf{4}} ; \eta\right)= & \left(\Delta\left(\mathbf{x}_{\mathbf{1}}, \mathbf{x}_{\mathbf{2}} ; \eta\right) \Delta\left(\mathbf{x}_{\mathbf{3}}, \mathbf{x}_{\mathbf{4}} ; \eta\right)-\right. \\
& -4 \int \frac{d^{3} \mathbf{k}}{2 \pi^{3}} \int \frac{d^{3} \mathbf{k}^{\prime}}{2 \pi^{3}}\left|v_{\mathbf{k}}\right|^{2}\left|v_{\mathbf{k}^{\prime}}\right|^{2} k^{2} k^{\prime 2} \epsilon^{2} \eta^{4} \times \\
& \left.\times \sin ^{2} \vartheta \sin ^{2} \vartheta^{\prime} \sin ^{2} \psi e^{i \mathbf{k} \cdot\left(\mathbf{x}_{\mathbf{1}}-\mathbf{x}_{\mathbf{2}}\right)} e^{i \mathbf{k}^{\prime} \cdot\left(\mathbf{x}_{\mathbf{3}}-\mathbf{x}_{\mathbf{4}}\right)}\right)+ \\
& +(2 \leftrightarrow 3)+(2 \leftrightarrow 4)
\end{aligned}
$$

where $v_{\mathbf{k}}$ is given in (4.7) and with, as in the previous section, $k_{\perp} \equiv k \sin \vartheta, k_{\perp}^{\prime} \equiv k^{\prime} \sin \vartheta^{\prime}$, and moreover $\mathbf{k}_{\perp} \cdot \mathbf{k}_{\perp}^{\prime} \equiv k_{\perp} k_{\perp}^{\prime} \cos \psi$. Notice that the same result holds for the correlation 
functions of the Fourier anti-transform of $\zeta_{\mathbf{k}}$, since the factor $\mathrm{Ha} / \dot{\phi}$ depends on $\eta$ only. Eq. (4.12) shows that, for late times $\eta \rightarrow 0$, well after horizon crossing for those scales which are of cosmological interests today, deviation from gaussianity due to the noncommutativity scale $\Lambda$ is suppressed by the factor $(k / a \Lambda)^{4}$. This is actually a consequence of the fact that in the comoving frame $\Theta^{12}$ scales as $a^{-2}$, so that its role at energy scales much smaller that $\Lambda$ is expected to be negligible. ${ }^{7}$ It is important to stress again that deviation from isotropy, encoded in the parameter $\alpha$, see eq. (4.7), is instead unaffected by the evolution after horizon crossing. Perturbation amplitudes keep in fact their momentum distribution at horizon crossing until they become again sub horizon sized at late times. In the following we neglect in (4.11) all contributions which vanish in the limit $\eta \rightarrow 0$.

Since we do not expect any sensible deviation from gaussianity, it remains true that all information on $\mathrm{CMB}$ temperature anisotropy is encoded in the correlation functions $\left\langle a_{l m}^{*} a_{l^{\prime} m^{\prime}}\right\rangle$. In the standard case one gets ${ }^{8}$

$$
\left\langle\zeta_{\mathbf{k}}^{*} \zeta_{\mathbf{k}^{\prime}}\right\rangle \propto\left|v_{k}\right|^{2} \delta^{3}\left(\mathbf{k}-\mathbf{k}^{\prime}\right)
$$

and for the coefficients of the CMB power spectrum

$$
\left\langle a_{l m}^{*} a_{l^{\prime} m^{\prime}}\right\rangle=C_{0}(l) \delta_{l l^{\prime}} \delta_{m m^{\prime}} .
$$

In this last expression, the uncorrelation for $l \neq l^{\prime}$ is simply a consequence of the rotational invariance of $\left|\zeta_{\mathbf{k}}\right|$ in eq. (4.13). In our case, from (4.11), we see that eq. (4.13) should be replaced by

$$
\begin{aligned}
\int \frac{d^{3} \mathbf{x}_{\mathbf{1}}}{(2 \pi)^{3 / 2}} & \frac{d^{3} \mathbf{x}_{\mathbf{2}}}{(2 \pi)^{3 / 2}} e^{i \mathbf{k} \cdot \mathbf{x}_{\mathbf{1}}} e^{-i \mathbf{k}^{\prime} \cdot \mathbf{x}_{\mathbf{2}}}\left\langle\zeta\left(\mathbf{x}_{\mathbf{1}}, \eta\right) * \zeta\left(\mathbf{x}_{\mathbf{2}}, \eta\right)\right\rangle \simeq \\
& \simeq\left\langle\zeta_{\mathbf{k}}^{*} \zeta_{\mathbf{k}^{\prime}}\right\rangle \propto\left|v_{\mathbf{k}}\right|^{2} \delta^{3}\left(\mathbf{k}-\mathbf{k}^{\prime}\right) \propto k^{-3}\left(1-\frac{3}{2} \epsilon^{2} \sin ^{2} \vartheta\right),
\end{aligned}
$$

with $1 / k, 1 / k^{\prime} \gg|\eta| \simeq 0$. Therefore we get

$$
\begin{aligned}
\left\langle a_{l m}^{*} a_{l^{\prime} m^{\prime}}\right\rangle \simeq C_{0}(l)\{ & \delta_{l, l^{\prime}} \delta_{m, m^{\prime}}\left[1+\frac{3}{2} \epsilon^{2}\left(\frac{(l+1)^{2}-m^{2}}{(2 l+1)(2 l+3)}+\frac{l^{2}-m^{2}}{(2 l-1)(2 l+1)}-1\right)\right]-(4 . \\
& \left.-\left(\frac{3 \epsilon^{2} \tilde{\mathcal{T}}_{l l^{\prime}}}{2(4 l-3)} \sqrt{\frac{\left((l-1)^{2}-m^{2}\right)\left(l^{2}-m^{2}\right)}{(2 l-1)(2 l+1)}} \delta_{l-2, l^{\prime}} \delta_{m, m^{\prime}}-l \leftrightarrow l^{\prime}\right)\right\},
\end{aligned}
$$

where

$$
\tilde{\mathcal{T}}_{l l^{\prime}} \equiv \frac{\int_{0}^{\infty} \frac{d k}{k} \mathcal{T}(k, l) \mathcal{T}\left(k, l^{\prime}\right)}{\int_{0}^{\infty} \frac{d k}{k} \mathcal{T}^{2}(k, l)} \sim 1,
$$

for scales of cosmological interest $l \geq 100$, and for $l^{\prime}=l \pm 2$.

\footnotetext{
${ }^{7}$ This result agrees with what has been obtained in one of the two scenarios considered in ref. [14], with $\Theta^{i j} \sim a^{-2}$. The same conclusion should also hold a fortiori in the other ansatz they consider (a step function behaviour for $\Theta^{\mu \nu}$ ) since, at the energy scale relevant for $\mathrm{CMB}, \Theta^{i j}=0$, and their $*$ product reduces to ordinary pointwise product.

${ }^{8}$ Hereafter we neglect numerical coefficients which factorize in the final expression (4.16).
} 
The correlation (4.16) between different multipoles $l$ and $l \pm 2$ is the main effect of the preferred direction singled out by $\Theta^{12}$ and, at least in principle, may be tested in future CMB experiments like PLANCK. As we already noticed, this effect is not diluted by the expansion, since it is related to momentum distribution of fluctuations at horizon crossing. Notice that the breaking of isotropy leads, in our model, to a quadrupole effect rather than, as one may have expected, to a dipole term. This is due to the fact that the first modifications induced by the $*$ product (2.6) are of quadratic order in $\Theta^{\mu \nu}$.

\section{Conclusions}

In this work we have discussed how cosmological perturbations may be affected by a modification of standard physics at short distances motivated by Noncommutative Geometry. These studies in the framework of inflationary cosmology provide a unique chance to test physical laws at very small scales, since the perturbations are generated as quantum fluctuations on sub horizon scales $\left(d<\left(10^{13} \mathrm{GeV}\right)^{-1}\right)$, and then stretched to cosmological sizes.

To study the evolution of perturbations during inflation, we have adopted a (nonassociative) covariant generalization of the $\star$ product used in flat spaces, characterized by a length scale $\Lambda^{-1}$ constant in physical coordinates. This parameter is related to the vacuum expectation value of the antisymmetric tensor $\Theta^{\mu \nu}$. At physical distances much greater than $\Lambda^{-1}$ this product reduces to the usual one, and standard physics is recovered. The deformed action for fluctuations has been studied at second order in a $\Theta$ perturbative expansion. The regime of validity of this analysis is carefully discussed in the paper, as well as associativity, which is preserved at this level of approximation. The main effect on fluctuations turns out to be a modified dispersion relation, which breaks isotropy.

Finally we have discussed how these effects may be possibly detected in the CMB anisotropy. While the gaussian character of the fluctuations is preserved, the particular direction taken by the nonvanishing $\left\langle\Theta^{i j}\right\rangle$ breaks isotropy. Since the main effect is quadratic in $\Theta^{\mu \nu}$, this leads to a quadrupole contribution to the CMB spectrum. The size of the quadrupole term is of order $(H / \Lambda)^{4}$, where $H$ is the Hubble parameter during inflation. Thus, it can be visible only provided the scale $\Lambda^{-1}$ is not much smaller than the size of the horizon during inflation. Forthcoming satellite experiments, with improved detection accuracy, as well as a wider sky coverage, may give a chance for detection of this small quadrupole contribution.

\section{Acknowledgments}

M.P. acknowledges Stefan Groot Nibbelink for useful discussions. The work of F.L. is supported in part by the Progetto di Ricerca di Interesse Nazionale SInteSi. M.P. is supported by the European Community's Human Potential Programme under contract HPRN-CT2000-00131 Quantum Spacetime, and under contracts HPRN-CT-2000-00148 and 00152. 


\section{References}

[1] V.S. Kaplunovsky, One loop threshold effects in string unification, Nucl. Phys. B 307 (1988) 145 hep-th/9205068, erratum, ibid. B382 (1992) 436.

[2] I. Antoniadis, A possible new dimension at a few TeV, Phys. Lett. B 246 (1990) 377.

[3] N. Arkani-Hamed, S. Dimopoulos and G.R. Dvali, The hierarchy problem and new dimensions at a millimeter, Phys. Lett. B 429 (1998) 263 hep-ph/9803315.

[4] R.H. Brandenberger and J. Martin, The robustness of inflation to changes in super-Planck-scale physics, Mod. Phys. Lett. A 16 (2001) 999 [astro-ph/0005432.

[5] J. Martin and R.H. Brandenberger, The trans-planckian problem of inflationary cosmology, Phys. Rev. D 63 (2001) 123501 [hep-th/0005209].

[6] R.H. Brandenberger, S.E. Joras and J. Martin, Trans-planckian physics and the spectrum of fluctuations in a bouncing universe, hep-th/0112122.

[7] J. Martin and R.H. Brandenberger, The Corley-Jacobson dispersion relation and trans-planckian inflation, Phys. Rev. D 65 (2002) 103514 hep-th/0201189.

[8] R.H. Brandenberger and J. Martin, On signatures of short distance physics in the cosmic microwave background, hep-th/0202142.

[9] J. Kowalski-Glikman, Testing dispersion relations of quantum kappa-Poincaré algebra on cosmological ground, Phys. Lett. B 499 (2001) 1 astro-ph/0006250.

[10] A. Kempf, Mode generating mechanism in inflation with cutoff, Phys. Rev. D 63 (2001) 083514 astro-ph/0009209.

[11] J.C. Niemeyer, Inflation with a high frequency cutoff, Phys. Rev. D 63 (2001) 123502 astro-ph/0005533.

[12] J.C. Niemeyer and R. Parentani, Trans-planckian dispersion and scale-invariance of inflationary perturbations, Phys. Rev. D 64 (2001) 101301 [astro-ph/0101451.

[13] A. Kempf and J.C. Niemeyer, Perturbation spectrum in inflation with cutoff, Phys. Rev. D 64 (2001) 103501 astro-ph/0103225.

[14] C.-S. Chu, B.R. Greene and G. Shiu, Remarks on inflation and noncommutative geometry, Mod. Phys. Lett. A 16 (2001) 2231 hep-th/0011241.

[15] R. Easther, B.R. Greene, W.H. Kinney and G. Shiu, Inflation as a probe of short distance physics, Phys. Rev. D 64 (2001) 103502 hep-th/0104102.

[16] R. Easther, B.R. Greene, W.H. Kinney and G. Shiu, Imprints of short distance physics on inflationary cosmology, hep-th/0110226.

[17] L. Mersini, M. Bastero-Gil and P. Kanti, Relic dark energy from trans-planckian regime, Phys. Rev. D 64 (2001) 043508 hep-ph/0101210.

[18] G. Amelino-Camelia and T. Piran, Cosmic rays and TeV photons as probes of quantum properties of space-time, Phys. Lett. B 497 (2001) 265 hep-ph/0006210.

[19] A. Mazumdar and M.M. Sheikh-Jabbari, Noncommutativity in space and primordial magnetic field, Phys. Rev. Lett. 87 (2001) 011301 hep-ph/0012363.

[20] S. Alexander and J. Magueijo, Non-commutative geometry as a realization of varying speed of light cosmology, hep-th/0104093. 
[21] S. Alexander, R. Brandenberger and J. Magueijo, Non-commutative inflation, hep-th/0108190.

[22] A. Kempf, G. Mangano and R.B. Mann, Hilbert space representation of the minimal length uncertainty relation, Phys. Rev. D 52 (1995) 1108 hep-th/9412167.

[23] A. Kempf and G. Mangano, Minimal length uncertainty relation and ultraviolet regularisation, Phys. Rev. D 55 (1997) 7909 hep-th/9612084.

[24] A. Connes, M.R. Douglas and A. Schwarz, Noncommutative geometry and matrix theory: compactification on tori, J. High Energy Phys. 02 (1998) 003 hep-th/9711162.

[25] N. Seiberg and E. Witten, String theory and noncommutative geometry, J. High Energy Phys. 09 (1999) 032 hep-th/9908142.

[26] F. Lizzi and R.J. Szabo, Duality symmetries and noncommutative geometry of string spacetimes, Commun. Math. Phys. 197 (1998) 667 hep-th/9707202.

[27] A. Connes, Noncommutative geometry, Academic Press, 1994.

[28] J. Madore, An introduction to noncommutative geometry and its physical applications, Cambridge University Press, 1999.

[29] G. Landi, An introduction to noncommutative spaces and their geometries, Springer Verlag, 1997.

[30] J.M. Gracia-Bondía, J.C. Várilly and H. Figueroa, Elements of noncommutative geometry, Birkhäuser, 2001.

[31] F. Bayen, M. Flato, C. Fronsdal, A. Lichnerowicz and D. Sternheimer, Deformation theory and quantization, 1. Deformations of symplectic structures, Ann. Phys. (NY) 111 (1978) 61.

[32] H.J. Groenewold, On the principles of elementary quantum mechanics, Physica 12 (1946) 405 .

[33] J.E. Moyal, Quantum mechanics as a statistical theory, Proc. Cambridge Phil. Soc. 45 (1949) 99.

[34] S. Doplicher, K. Fredenhagen and J. E. Roberts, The quantum structure of space-time at the Planck scale and quantum fields, Commun. Math. Phys. 172 (1995) 187.

[35] T. Filk, Divergencies in a field theory on quantum space, Phys. Lett. B 376 (1996) 53.

[36] J.C. Várilly and J.M. Gracia-Bondía, On the ultraviolet behaviour of quantum fields over noncommutative manifolds, Int. J. Mod. Phys. A 14 (1999) 1305 hep-th/9804001.

[37] S. Minwalla, M. Van Raamsdonk and N. Seiberg, Noncommutative perturbative dynamics, 刃. High Energy Phys. 02 (2000) 020 hep-th/9912072.

[38] R.J. Szabo, Quantum field theory on noncommutative spaces, hep-th/0109162.

[39] M.R. Douglas and N.A. Nekrasov, Noncommutative field theory, Rev. Mod. Phys. 73 (2002) 977 hep-th/0106048.

[40] A. Konechny and A. Schwarz, Introduction to M(atrix) theory and noncommutative geometry, Phys. Rept. 360 (2002) 353 hep-th/0012145.

[41] A. Konechny and A. Schwarz, Introduction to M(atrix) theory and noncommutative geometry, part II, Phys. Rept. 360 (2002) 353 hep-th/0107251. 
[42] N. Seiberg, L. Susskind and N. Toumbas, Strings in background electric field, space/time noncommutativity and a new noncritical string theory, J. High Energy Phys. 06 (2000) 021 hep-th/0005040.

[43] J. Gomis and T. Mehen, Space-time noncommutative field theories and unitarity, Nucl. Phys. B 591 (2000) 265 hep-th/0005129.

[44] N. Seiberg, L. Susskind and N. Toumbas, Space/time non-commutativity and causality, J. High Energy Phys. 06 (2000) 044 hep-th/0005015.

[45] R. Gopakumar, J.M. Maldacena, S. Minwalla and A. Strominger, S-duality and noncommutative gauge theory, J. High Energy Phys. 06 (2000) 036 hep-th/0005048.

[46] D. Bahns, S. Doplicher, K. Fredenhagen and G. Piacitelli, On the unitarity problem in space/time noncommutative theories, Phys. Lett. B 533 (2002) 178 hep-th/0201222.

[47] M. Kontsevich, Deformation quantization of Poisson manifolds, 1, q-alg/9709040.

[48] J.M. Gracia-Bondía, F. Lizzi, G. Marmo and P. Vitale, Infinitely many star products to play with, J. High Energy Phys. 04 (2002) 026 hep-th/0112092.

[49] S. Gutt, An explicit *-star product on the cotangent bundle of a Lie group, Lett. Math. Phys. 7 (1983) 249.

[50] J. Grabowski, Abstract Jacobi and Poisson structures: quantization and star products, J. Geom. Phys. 9 (1992) 45.

[51] J. Madore, S. Schraml, P. Schupp and J. Wess, Gauge theory on noncommutative spaces, Eur. Phys. J. C 16 (2000) 161 hep-th/0001203.

[52] L. Cornalba and R. Schiappa, Nonassociative star product deformations for D-brane worldvolumes in curved backgrounds, Commun. Math. Phys. 225 (2002) 33 hep-th/0101219.

[53] J.A. Peacock, Cosmological physics, Cambridge University Press, Cambridge 1999.

[54] V.F. Mukhanov, H.A. Feldman and R.H. Brandenberger, Theory of cosmological perturbations. part 1. classical perturbations. part 2. quantum theory of perturbations. part 3. extensions, Phys. Rept. 215 (1992) 203.

[55] J.M. Bardeen, Gauge invariant cosmological perturbations, Phys. Rev. D 22 (1980) 1882.

[56] V.F. Mukhanov, Quantum theory of gauge invariant cosmological perturbations, Sov. Phys. JETP 67 (1988) 1297 [Zh. Eksp. Teor. Fiz. 94N7 (1988) 1].

[57] D.H. Lyth and A. Riotto, Particle physics models of inflation and the cosmological density perturbation, Phys. Rept. 314 (1999) 1 hep-ph/9807278. 